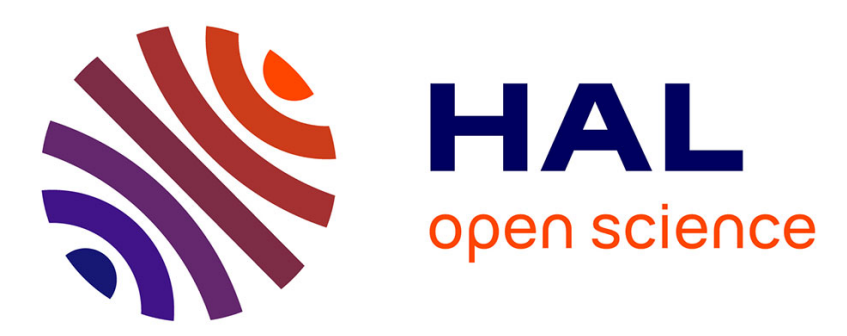

\title{
Oser la conception innovante pour une industrie de la mode et du luxe durable et responsable Benjamin Cabanes
}

\section{To cite this version:}

Benjamin Cabanes. Oser la conception innovante pour une industrie de la mode et du luxe durable et responsable. Entreprendre \& Innover, 2021, nº 46 (3), pp.42-55. 10.3917/entin.046.0042 . hal03207274

\section{HAL Id: hal-03207274 \\ https://hal.science/hal-03207274}

Submitted on 8 Jun 2021

HAL is a multi-disciplinary open access archive for the deposit and dissemination of scientific research documents, whether they are published or not. The documents may come from teaching and research institutions in France or abroad, or from public or private research centers.
L'archive ouverte pluridisciplinaire HAL, est destinée au dépôt et à la diffusion de documents scientifiques de niveau recherche, publiés ou non, émanant des établissements d'enseignement et de recherche français ou étrangers, des laboratoires publics ou privés. 
Pour citer cet article : Cabanes, B. (2021). Oser la conception innovante pour une industrie de la mode et du luxe durable et responsable. Entreprendre \& Innover, 3(3), 42-55. https://doi.org/10.3917/entin.046.0042

\section{Oser la conception innovante pour une industrie de la mode et du luxe durable et responsable}

\section{Résumé :}

L'industrie de la mode et du luxe est aujourd'hui la deuxième industrie la plus polluante au monde. Elle génère à elle seule $10 \%$ du total des gaz à effets de serre. Face à ce constat, ce secteur industriel se doit d'innover et de tendre vers un développement plus durable et responsable. Quelles sont les stratégies d'innovation pour transformer les contraintes environnementales et sociétales en opportunités d'affaires et en forces stratégiques ? Cet article met en évidence une approche originale qui vise à explorer collectivement et systématiquement les enjeux et défis du développement durable propres au secteur de la mode et du luxe et à repérer des voies d'innovation à exploiter. II s'agit de concevoir la stratégie d'innovation comme une activité de conception innovante, c'est-à-dire une approche ex ante qui a pour objectif d'expliciter et de formaliser des attentes, des besoins et des réponses autour de concepts, d'objets et de services «désirables » et de repenser ainsi l'utilité économique et sociale des produits et des activités de la mode et du luxe en fonction de leur impact social et environnemental.

\section{Points-forts :}

- L'industrie de la mode et du luxe est l'une des industries les plus polluantes au monde, par conséquent, le développement durable est devenu aujourd'hui un champ d'innovation incontournable que les entreprises du secteur doivent explorer afin d'inventer de nouvelles solutions soutenables et durables.

- Il existe des stratégies d'innovation ex ante qui visent à réduire et à compenser les externalités négatives de ce secteur industriel mais elles ne sont pas à la hauteur des enjeux et des défis rencontrés. 
Pour citer cet article : Cabanes, B. (2021). Oser la conception innovante pour une industrie de la mode et du luxe durable et responsable. Entreprendre \& Innover, 3(3), 42-55. https://doi.org/10.3917/entin.046.0042

- Certaines marques mettent en place des stratégies originales qui ne se limitent pas à la réduction des impacts négatifs mais qui proposent de nouvelles stratégies aux impacts positifs.

- La méthode KCP permet une vision renouvelée de la stratégie d'innovation fondée sur une démarche collective d'exploration systématique de champs d'innovation.

La mode, et plus particulièrement l'industrie du textile, est aujourd'hui reconnue comme étant le secteur industriel le plus polluant au monde, après l'industrie du pétrole. Selon l'Ademe (Agence de l'environnement et de la maîtrise de l'énergie), l'industrie du textile génère à elle seule $10 \%$ du total des gaz à effets de serre et consomme $25 \%$ des produits chimiques produits chaque année. Cependant, l'impact de l'industrie de la mode sur l'environnement ne se limite pas à la production de gaz à effets de serre ou à son empreinte carbone. En effet, le secteur industriel de la mode a un impact considérable sur la consommation d'eau. Par exemple, la production d'un jean nécessite environ 11000 litres d'eau, soit l'équivalent de 300 douches $^{1}$. Or chaque année en France, 90 millions de jeans sont vendus. Le rapport Pulse of the Fashion Industry? souligne que l'industrie de la mode consomme plus de 79 milliards de mètres cubes d'eau à l'échelle mondiale, alors même que la production du textile s'effectue principalement dans des zones de forte sècheresse. L'eutrophisation de l'eau, c'est-à-dire la modification chimique de l'eau à cause des polluants qui y sont déversés, est aussi un fléau. En Chine, $70 \%$ des

\footnotetext{
1 Barroux R. (2017, Juin). "La mode se penche sur son poids environnemental ". Le Monde. https://www.lemonde.fr/planete/article/2017/06/10/la-mode-se-penche-sur-son-poidsenvironnemental_5142067_3244.html 2 Kerr, J., \& Landry, J. (2017). Pulse of the Fashion Industry. Global Fashion Agenda and the Boston Consulting Group.
} 
Pour citer cet article : Cabanes, B. (2021). Oser la conception innovante pour une industrie de la mode et du luxe durable et responsable. Entreprendre \& Innover, 3(3), 42-55. https://doi.org/10.3917/entin.046.0042

cours d'eau sont pollués à cause de l'industrie textile ${ }^{3}$. Un autre rapport ${ }^{4}$, insiste tout particulièrement sur la production de déchets et l'absence de filière de recyclage dans l'industrie de la mode et de l'habillement. Le rapport souligne que sur les 53 millions de tonnes de matières produites pour la fabrication des vêtements, moins de $15 \%$ sont recyclés pour la confection de nouveaux vêtements.

Les externalités négatives ${ }^{5}$ de l'industrie de la mode et du luxe ne concernent pas seulement la dégradation de l'environnement. Les conséquences sociales et sociétales sont toutes aussi dramatiques. En 2013, le Rana Plaza au Bengladesh, immeuble qui abritait des ateliers de fabrication de vêtement, s'écroulait, faisant 1135 morts et plus de 2000 blessés. Parmi les clients de ces ateliers figuraient les plus grandes marques occidentales parmi lesquelles El Corte Inglès, Auchan, Benetton et Mango. Cet événement dramatique a mis en lumière les conditions de travail déplorables des ouvriers et l'insalubrité du bâtiment. La fondation Ellen MacArthur souligne que plus de $50 \%$ des ouvriers du textile ne sont pas rémunérés selon le salaire minimum légal de leur pays. L'écart de salaire entre les hommes et les femmes est souvent considérable. Au Pakistan, dans l'industrie du vêtement, 87\% des femmes et $27 \%$ des hommes sont payés en dessous du minimum légal. Enfin, il est aussi important de souligner que l'industrie de la mode est très souvent accusée de favoriser l'anorexie chez les jeunes femmes en raison de la promotion d'un idéal de corps féminin irréaliste.

\footnotetext{
${ }_{3}^{3}$ "Quel est l'impact de l'industrie textile sur l'environnement ?», Huffington Post France. https://www.huffingtonpost.fr/2015/11/29/impact-textile-environnem_n_8663002.html

${ }^{4}$ Ellen MacArthur Foundation (2017). New textiles economy: redesigning fashion's future, p. 150.

5 Une externalité négative désigne le fait que la production ou la consommation d'un bien a une influence négative sur le bien-être d'une tierce partie (par exemple, la pollution, le bruit, le changement climatique).
} 
Pour citer cet article : Cabanes, B. (2021). Oser la conception innovante pour une industrie de la mode et du luxe durable et responsable. Entreprendre \& Innover, 3(3), 42-55. https://doi.org/10.3917/entin.046.0042

Suite à ce constat accablant, des réponses mises au service du développement durable s'imposent comme un impératif stratégique et éthique à toutes les parties prenantes du secteur: producteurs, marques, créateurs, façonniers, fabricants, fournisseurs et distributeurs, consommateurs sont tous concernés. Mais comment mettre en œuvre une stratégie favorisant une industrie de la mode plus durable et responsable ? Quelles sont les stratégies d'innovation les plus performantes ? Comment concevoir de nouvelles solutions innovantes?

\section{Des initiatives multiples mais pas de vision stratégique partagée}

\section{par l'ensemble des acteurs}

Pour les industriels du secteur de la mode et du luxe, ces externalités négatives sont connues et identifiées depuis longtemps. De leur côté, les consommateurs sont de plus en plus attentifs à l'impact environnemental et social des produits qu'ils achètent. Par conséquent, innover pour favoriser une mode plus durable devient un enjeu de performance et de compétitivité indispensable. Parmi les géants mondiaux de la mode et du luxe, en 2012, le groupe français Kering (Gucci, Saint Laurent, Balenciaga, etc.) a été l'un des premiers à communiquer ses objectifs de développement durable. En 2014, dans un rapport d'avancement $\underline{6}$, le groupe s'engageait notamment à ce que $100 \%$ des peaux précieuses et des fourrures utilisées dans les produits Kering proviennent d'élevages contrôlés ou encore à réduire de $25 \%$ la consommation d'eau, les déchets et les émissions de CO2 d'ici 2106. De son côté, LVMH, le leader mondial du luxe, publie également un rapport

\footnotetext{
6 Disponible en ligne sur le site internet de Kering. https://www.kering.com/fr/actualites/publie-unrapport-progres-ses-objectifs-developpement-durable-2016 consulté en novembre 2019
} 
Pour citer cet article : Cabanes, B. (2021). Oser la conception innovante pour une industrie de la mode et du luxe durable et responsable. Entreprendre \& Innover, 3(3), 42-55. https://doi.org/10.3917/entin.046.0042

d'activité $^{7}$ concernant ses engagements en matière de développement durable. Si les intentions sont louables, force est de constater que les stratégies affichées ne sont pas des plus innovantes. Finalement, elles se résument à réduire et compenser les impacts négatifs produits par l'entreprise. Dans son rapport d'activité de 2014, Kering explique que le groupe a compensé ses émissions de CO2 en achetant des crédits carbone pour $81 \%$ auprès de Wildlife Works Carbon, entreprise dont Kering est actionnaire depuis 2012. Se limiter à compenser l'impact environnemental négatif par l'intermédiaire d'une organisation dont on est actionnaire, n'est pas le meilleur signal envoyé ni une stratégie très innovante. Finalement, l'industrie du luxe se retrouve prise dans un dilemme : continuer à fonder son identité sur un savoir-faire d'excellence souvent secret, un fort imaginaire symbolique ou accepter d'être plus transparente au risque de se montrer imparfaite et peu inefficace en matière de développement durable.

De nouvelles initiatives ont néanmoins émergé ces dernières années. La marque de sneakers Veja, par exemple, propose une approche singulière dans le secteur de la chaussure. Veja travaille, sans intermédiaires, avec des producteurs de coton bio au Brésil, et les relations commerciales suivent les principes du commerce équitable. La marque assure acheter le coton deux fois plus cher que le prix du marché. Elle a confié sa logistique à Ateliers Sans Frontières, association de réinsertion par le travail en France. Enfin, la marque a adopté une communication entièrement

7 Disponible en ligne sur le site internet de LVMH. https://r.lvmhstatic.com/uploads/2014/07/lvmh environment 2017fr.pdf consulté en novembre 2019 
Pour citer cet article : Cabanes, B. (2021). Oser la conception innovante pour une industrie de la mode et du luxe durable et responsable. Entreprendre \& Innover, 3(3), 42-55. https://doi.org/10.3917/entin.046.0042

transparente $^{8}$ et n'hésite pas à expliciter les limites de son modèle de développement.

Toujours, dans le domaine de la chaussure, on peut également citer l'exemple de la marque Faguo dont la stratégie de communication repose sur un « concept» simple : pour un produit acheté, un arbre est planté en France. A ce jour, l'entreprise revendique plus d'un million d'arbres plantés.

Dans le domaine du textile, la marque Patagonia s'est érigée en modèle de «l'anti fast-fashion $^{9}$ » en incitant ses clients à réparer et garder leurs vêtements plutôt qu'à les remplacer. Patagonia, marque qui reverse $1 \%$ de ses ventes à des associations de protection de l'environnement, est certifiée «Benefit Corporation » ${ }^{10}$ et revendique comme «raison d'être » la fabrication de produits de haute qualité causant le moins d'impacts négatifs à l'environnement. Concilier la performance économique et la performance environnementale et sociale de l'entreprise, c'est aussi la vision défendue par la marque Eileen Fisher. Pour cette entreprise le développement durable ne doit pas reposer sur des initiatives ponctuelles et particulières ${ }^{11}$, mais doit être au fondement des stratégies de développement des entreprises. Ces visions stratégiques sont en rupture avec les stratégies des grands groupes de luxe, précédemment exposées. Pour ces jeunes marques, il ne s'agit pas seulement de compenser ou de réduire des impacts négatifs mais de considérer les

\footnotetext{
${ }^{8}$ Les coûts des matières premières et de production sont affichés sur le site internet de la marque. D'autre part, les contrats avec les producteurs de coton bio et les résultats des tests chimiques sont en accès libre sur le site internet.

9 Le «fast fashion» est un concept anglo-saxon qui désigne le renouvellement permanent et extrêmement rapide des collections d'articles de mode afin de favoriser le renouvellement de la garderobe des clients.

10 Certification octroyée aux entreprises à but lucratif répondant à des exigences sociétales et environnementales.

11 Par exemple, réduction des émissions de $\mathrm{CO} 2$, recyclage, etc.
} 
Pour citer cet article : Cabanes, B. (2021). Oser la conception innovante pour une industrie de la mode et du luxe durable et responsable. Entreprendre \& Innover, 3(3), 42-55. https://doi.org/10.3917/entin.046.0042

enjeux environnementaux et sociaux comme étant des axes stratégiques de développement. Parallèlement à ces marques récentes désireuses de changer les pratiques de la mode, on peut aussi noter l'émergence de plusieurs initiatives collectives qui invitent à repenser le modèle de la mode (Sustainable Apparel Coalition, Better Work, Better Cotton Initiative, etc.). En 2015, la créatrice de tendances néerlandaise Li Edelkoort, a publié un manifeste, intitulé Anti-fashion: A manifesto for the next decade ${ }^{12}$, dans lequel elle déplore le manque d'innovation conceptuelle dans l'industrie de la mode. Dans ce manifeste, l'auteure pointe du doigt les limites et l'obsolescence du système actuel de la mode, tant au niveau écologique que sociétal ou créatif. Li Edelkoort défend l'idée selon laquelle l'éducation des futurs créateurs permettra de changer le système de l'intérieur et favorisera l'émergence d'une mode plus soucieuse de justice et plus respectueuse de l'environnement.

Pour Godart et Seong ${ }^{13}$, trois principaux scénarios sont susceptibles de rendre l'industrie de la mode et du luxe plus durable. Le premier scénario résulterait d'un changement institutionnel favorisant l'adoption d'un modèle de développement de type slow fashion remplaçant ainsi le fast fashion ${ }^{14}$ (diminution du nombre de collection, rallongement des cycles de collections, incitation au non-renouvellement des articles de mode). Le second scénario permettrait un changement des processus de conception et de production (utilisation de nouveaux matériaux durables, réduction des déchets à chaque étape de fabrication, développement de filière de

\footnotetext{
12 https://www.edelkoort.com/fr/2015/09/anti_fashion-manifesto/

${ }^{13}$ Godart, F. \& Seong, S. (2014). "Is sustainable luxury fashion possible?"in M. A. Gardetti \& A. L. Torres (eds.), Sustainable Luxury: Managing Social and Environmental Performance in Iconic Brands, 12-27. Sheffield Greenleaf Publishing.

14 Le concept de slow fashion s'oppose à celui de fast fashion et incite à une consommation plus durable dans le temps des articles de mode.
} 
Pour citer cet article : Cabanes, B. (2021). Oser la conception innovante pour une industrie de la mode et du luxe durable et responsable. Entreprendre \& Innover, 3(3), 42-55. https://doi.org/10.3917/entin.046.0042

recyclage). Enfin, le troisième scénario se fonderait sur l'établissement de nouvelles contraintes réglementaires imposées par l'État ou les industriels. Cependant, n'existerait-il pas d'autres scénarios possibles ? Comment proposer de nouveaux scénarios ou de nouvelles pistes d'innovation favorisant l'émergence d'une mode durable et responsable?

\section{Le développement durable : un projet stratégique et un champ d'innovation à explorer}

Ces situations et initiatives multiples illustrent la façon dont l'identité des produits et les attentes sociales évoluent et se transforment (coûts, modes de production et de distribution, transparence des processus de fabrication, valorisation des produits, consommation, image de marque). Elles nous invitent à nous interroger sur l'impact des enjeux du développement durable sur l'industrie de la mode et sur les stratégies des entreprises: comment l'émergence de nouvelles attentes sociales et environnementales peuvent-elles stimuler les stratégies des entreprises ? Comment les enjeux du développement durable sont susceptibles de redistribuer l'équilibre des positions au sein d'une industrie ? Comment les entreprises peuvent-elles proposer de nouvelles propositions de valeur dans leurs offres de produits ? Comment la transformation des produits et services peut-elle constituer de nouvelles relations marques/clients ? Pour Acquier ${ }^{15}$, ces questions sont centrales pour penser le lien entre le développement durable et la stratégie des entreprises. Cependant, pour l'auteur, les cadres théoriques existants dans le domaine du développement durable et de la RSE ${ }^{16}$ abordent peu ces questions.

\footnotetext{
${ }^{15}$ Acquier., A. (2008). " Développement durable et management stratégique : piloter un processus de transformation de la valeur ».17e Conférence Internationale de l'AIMS.

${ }^{16}$ Responsabilité Sociale de l'Entreprise.
} 
Pour citer cet article : Cabanes, B. (2021). Oser la conception innovante pour une industrie de la mode et du luxe durable et responsable. Entreprendre \& Innover, 3(3), 42-55. https://doi.org/10.3917/entin.046.0042

Une première approche théorique, fondée sur la théorie des parties prenantes $(\text { Stakeholders })^{17}$, consiste à penser et analyser l'entreprise « comme une constellation d'intérêts coopératifs et compétitifs »18. Dans cette approche théorique, la finalité économique de l'entreprise n'est pas remise en cause, mais l'entreprise se doit de reconnaître un certain nombre de responsabilités à l'égard de la société et en particulier de ses parties prenantes (partenaires industriels, consommateurs, pouvoir public, ONG, etc.). Pour conserver sa capacité d'action, l'entreprise doit maintenir un ensemble de bonnes relations avec l'ensemble de ses parties prenantes, dont les intérêts peuvent être différents ${ }^{19}$. Dans cette perspective, les enjeux du développement durable sont interprétés et appréhendés en relation avec les préoccupations et attentes des parties prenantes ${ }^{20}$. En se focalisant sur les relations avec les acteurs externes de l'entreprise et en adoptant une représentation essentiellement politique de la firme, ce courant de recherche reste cependant perfectible pour l'analyse des stratégies de développement de produits et services. Pour Acquier et Aggeri21, l'approche des parties prenantes n'interroge pas les processus créatifs, les stratégies de développement de produits et les processus d'innovation au sein de l'entreprise. Pourtant, ces aspects semblent fondamentaux pour aborder les enjeux du développement durable au sein des entreprises.

\footnotetext{
17 Freeman R.E. (1984). Strategic Management: A Stakeholder Approach, Pitman. Aggeri, F., Pezet, E., Abrassart, C., \& Acquier, A. (2005). Organiser le développement durable. Vuibert.

18 Martinet, A. C., \& Reynaud, E. (2015). Shareholders, stakeholders et stratégie". Revue française de gestion, 41(253), 297-3.

${ }_{19}$ Gherra, S. (2010). "Stratégies de développement durable. Combiner les parties prenantes et les ressources et compétences de l'entreprise ». Revue française de gestion, 5(204), 141-153.

20 Allix-Desfautaux, E., \& Makany, L. G. D. (2015). "Développement durable et gestion d'une entreprise : croisements fertiles ". Management Avenir, (7), 15-36.

${ }^{21}$ Acquier, A., \& Aggeri, F. (2007). « Une généalogie de la pensée managériale sur la RSE ». Revue française de gestion, 11(180), 131-157.
} 
Pour citer cet article : Cabanes, B. (2021). Oser la conception innovante pour une industrie de la mode et du luxe durable et responsable. Entreprendre \& Innover, 3(3), 42-55. https://doi.org/10.3917/entin.046.0042

Une autre approche théorique consiste à considérer le développement durable comme étant un enjeu d'innovation stratégique pour les entreprises. Pour Michael Porter, les questions environnementales ${ }^{22}$ et les enjeux de responsabilité sociale de l'entreprise ${ }^{23}$ sont interdépendants et orientent la stratégie de l'entreprise. Michael Porter s'oppose à l'idée que la réglementation environnementale ajoute des contraintes supplémentaires pour les entreprises et affecte négativement leur compétitivité. Pour cet auteur, les contraintes environnementales et sociales contribuent à la fois à la réduction des externalités négatives et à la compétitivité des entreprises, c'est ce que l'on appelle «l' hypothèse Porter »24. En effet, ce dernier défend l'idée selon laquelle les contraintes poussent les organisations à innover, à remettre en question leurs modèles de développement initial et leur permettent ainsi d'obtenir des gains de productivité et par conséquent des avantages compétitifs nouveaux. Cependant, comme le remarque Franck Aggeri25, cette conception « gagnant-gagnant » ne propose qu'une vision appauvrie du processus d'innovation. L'enjeu consiste dans ce cas à uniquement repérer des opportunités préexistantes, quitte à les intégrer ensuite à la stratégie de l'entreprise.

II existe néanmoins une autre approche possible qui consiste à orienter la stratégie d'innovation vers l'exploration de nouveaux champs d'innovation, c'est-à-dire des domaines où s'exerce un travail d'apprentissage, de repérage de potentielles nouvelles valeurs, de création de nouveaux concepts de produits et de construction

\footnotetext{
22 Porter, M. E., \& Van der Linde, C. (1995). "Toward a new conception of the environmentcompetitiveness relationship". Journal of economic perspectives, 9(4), 97-118.

23 Porter, M. E., \& Kramer, M. R. (2006). "Strategy and society: The link between competitive advantage and corporate social responsibility". Harvard business review, 84(12), 78-92.

${ }^{24}$ Ambec, S., \& Lanoie, P. (2008). "Does it pay to be green? A systematic overview". Academy of management perspectives, 22(4), 45-62.

${ }^{25}$ Aggeri, F. (2011). "Le développement durable comme champ d'innovation ». Revue française de gestion, (6), 87-106.
} 
Pour citer cet article : Cabanes, B. (2021). Oser la conception innovante pour une industrie de la mode et du luxe durable et responsable. Entreprendre \& Innover, 3(3), 42-55. https://doi.org/10.3917/entin.046.0042

de nouvelles connaissances ${ }^{26}$. Faire du développement durable un champ d'innovation consiste alors à considérer cet enjeu comme étant un espace d'invention original pour la création de nouvelles connaissances et de nouvelles solutions inédites. Cette approche se fonde sur le fait que l'économie contemporaine impose aux entreprises des situations complexes d'où les futurs produits et services émergeront et se commercialiseront sur des marchés encore inexistants alors même que les compétences pour les concevoir ne sont pas encore disponibles. Par conséquent, l'enjeu est d'orienter les processus d'innovation vers une activité collective de conception innovante, c'est-à-dire une approche dont l'objectif est d'explorer un champ d'innovation pour repérer, expliciter et formaliser des attentes, des besoins et des réponses en les cristallisant autour de concepts, d'objets et de services « désirables »27, mais dont le potentiel de valeur reste à construire.

\section{La conception innovante : un cadre d'analyse pour explorer collectivement des champs d'innovation?}

La notion d'innovation est couramment définie comme une invention qui rencontre un marché. Cependant, cette définition est problématique pour deux raisons. Premièrement, elle fait de l'innovation un jugement et une évaluation ex post d'un produit existant, ignorant ainsi la capacité d'action à innover ex ante. Deuxièmement, elle favorise une définition singulière de l'innovation, ce qui a pour conséquence de masquer le caractère systémique de l'innovation (apprentissage, ressources, action

\footnotetext{
${ }^{26}$ Le Masson, P., Hatchuel, A., \& Weil, B. (2014). Théorie, méthodes et organisations de la conception. Presses des Mines.

27 Le Masson, P., Weil, B., \& Hatchuel, A. (2006). Les processus d'innovation : Conception innovante et croissance des entreprises. Lavoisier.
} 
Pour citer cet article : Cabanes, B. (2021). Oser la conception innovante pour une industrie de la mode et du luxe durable et responsable. Entreprendre \& Innover, 3(3), 42-55. https://doi.org/10.3917/entin.046.0042

collective, création de connaissance, diffusion de l'innovation, etc. $)^{28}$. Pour ces raisons, il est préférable de s'intéresser aux capacités de conception et à l'analyse des processus de l'action collective qui peuvent conduire à l'innovation.

Dans cette perspective, Le Masson et al. ${ }^{29}$ distinguent deux régimes de conception selon le mode de raisonnement de conception mobilisé, la forme d'organisation collective mobilisée et la logique de performance: la conception réglée et la conception innovante. La conception réglée a pour objectif d'améliorer, de manière continue, les produits et services existants. Les compétences et connaissances mobilisées sont connues et mobilisable par l'entreprises. Dans un régime de conception réglée, l'identité des objets, les « dominant designs »30 et les modèles économiques sont préservés. En revanche, la conception innovante caractérise un processus de conception ayant pour objectif de générer de nouvelles connaissances, de revisiter l'identité des objets et de créer de nouvelles offres de valeur. II n'est donc pas possible de déterminer à l'avance les ressources et les compétences nécessaires au pilotage du processus de conception. En conception innovante, l'enjeu consiste à générer simultanément de nouvelles lignées de concepts (potentiels nouveaux produits et services) et de nouvelles connaissances. Ce raisonnement de conception innovante a été modélisé par Armand Hatchuel et Benoit Weil ${ }^{31}$, et fait l'objet d'une théorie formelle, dite théorie $\mathrm{C}-\mathrm{K}^{32}$.

\footnotetext{
28 Archambault, V., \& Nakhla, M. (2015). «La conception innovante, un enjeu majeur pour l'environnement ». Sciences Eaux Territoires, (2), 4-9.

29 Op. cit.

30 Désigne les principales caractéristiques et propriétés qui permettent d'identifier un objet ou un produit.

31 Professeurs à MINES ParisTech, chaire Théorie et Méthodes de la Conception Innovante.

32 Le Masson, P., Hatchuel, A., \& Weil, B. (2018). Théorie CK-Fondements et implications d'une théorie de la conception. Technique de l'ingénieur.
} 
Pour citer cet article : Cabanes, B. (2021). Oser la conception innovante pour une industrie de la mode et du luxe durable et responsable. Entreprendre \& Innover, 3(3), 42-55. https://doi.org/10.3917/entin.046.0042

\section{La théorie C-K}

La théorie $\mathrm{C}-\mathrm{K}^{33}$ est une théorie de la conception et du raisonnement de conception issue d'observations et de nombreux travaux empiriques effectués dans le domaine de l'industrie. Le principe de la théorie $\mathrm{C}-\mathrm{K}$ repose sur la distinction entre deux espaces en constante expansion : l'espace des concepts (C) qui regroupe les propositions dites indécidables, imaginaires et inconnues; l'espace des connaissances (K pour Knowledge) qui regroupe l'ensemble des savoirs, connaissances et compétences connues et validées.

La théorie C-K stipule que l'activité de conception est un processus au cours duquel un concept engendre d'autres concepts et induit la création de nouvelles connaissances. Ces nouvelles connaissances produisent, à leur tour, de nouvelles connaissances et modifient les concepts initiaux.

${ }^{33}$ Le Masson, P., Mcmahon, C. (2016). « Armand Hatchuel et Benoit Weil La théorie C-K, un fondement formel aux théories de l'innovation »in Les grands auteurs du management de l'innovation et de la créativité. Editions Management et Société, pp. 588-613. 
Pour citer cet article : Cabanes, B. (2021). Oser la conception innovante pour une industrie de la mode et du luxe durable et responsable. Entreprendre \& Innover, 3(3), 42-55. https://doi.org/10.3917/entin.046.0042

\begin{tabular}{|c|}
\hline $\begin{array}{l}\text { Passer d'un espace à l'autre permet de rendre compte d'un double processus } \\
\text { d'expansion }{ }^{34} \text { : les connaissances permettent d'étendre les concepts (c'est-à-dire } \\
\text { d'ajouter au fur et à mesure de nouvelles propriétés aux concepts) et les nouveaux } \\
\text { concepts entraînent l'expansion des connaissances. L'expansion conjointe de ces } \\
\text { deux espaces engendre des objets inconnus à partir de faits connus. Par } \\
\text { conséquent, le cadre conceptuel de la théorie C-K permet d'appréhender la } \\
\text { nouveauté puisqu'il permet de distinguer l'état de l'art (connaissance disponible } \\
\text { dans l'espace K) et de faire émerger de nouveaux concepts (espace C). Enfin, } \\
\text { l'arborescence de l'espace C permet de mettre en évidence un ensemble } \\
\text { d'« inconnues désirables » constituant autant de stratégies d'innovation } \\
\text { alternatives à affiner ou à concrétiser. }\end{array}$ \\
\hline
\end{tabular}

\footnotetext{
${ }^{34}$ Hatchuel, A., Le Masson, P., Weil, B. (2017). « Comprendre et soutenir l'innovation contemporaine :
} théorie de la conception et métabolisme des nouveaux collectifs ». La lettre de l'InSHS. 


\section{Une recherche intervention pour accompagner une démarche de conception innovante dans l'industrie de la mode et du luxe}

Dans le cadre du programme CARATS ${ }^{35}$, une équipe de chercheurs de la chaire TMCl (Théorie et Méthodes de la Conception Innovante) de MINES ParisTech a conduit une recherche-intervention pour accompagner l'exploration collective du champ d'innovation suivant: une industrie de la mode et du luxe durable et responsable.

Pour accompagner cette démarche de conception innovante, l'équipe de chercheur s'est appuyé sur la méthode KCP (Knowledge - Concepts - Proposals). La méthode $\mathrm{KCP}^{36}$ est un outil de conception innovante issus de la théorie C-K. Son objectif est d'accompagner un collectif d'acteurs dans l'exploration d'un champ d'innovation et l'élaboration de stratégies d'innovation. Cette méthodologie a été mobilisée dans plusieurs projets de recherche-intervention et dans divers secteurs industriels ${ }^{37}$. La démarche KCP se décompose en trois phases distinctes (figure ci-dessous). Une phase de mutualisation intensive de connaissances (Phase K), ayant pour objectif de rendre possible un travail collectif sur un champ d'innovation particulier. Une phase d'exploration et de développement de nouveaux concepts (Phase C). Cette phase a pour but d'identifier de nouvelles voies d'innovation et de remettre en question

\footnotetext{
${ }^{35}$ Programme de recherche dédié à la filière mode et luxe (Instituts Carnot MINES, MICA et Ingénierie@Lyon). Voir Cabanes B. (2019). Innovation et recherche dans la mode et le luxe : exploration de champs d'innovation et programmes de recherche. [Rapport de recherche] Mines ParisTech - PSL University.

${ }^{36}$ Agogué, M., Arnoux, F., Brown, I., \& Hooge, S. (2013). Introduction à la Conception Innovante : éléments théoriques et pratiques de la théorie CK. Presses des MINES.

${ }^{37}$ Labatut, J., \& Hooge, S. (2016). « Renouveler la gestion de ressources communes par la conception innovante ? Le cas d'une race locale au Pays basque ». Natures Sciences Sociétés, 24(4), 319-330. Lageat, E., \& Lafon, D. (2017). « La théorie CK, cadre « naturel » pour innover en politique ? » in Entreprendre Innover, (1), 51-60. Laousse, D., \& Hooge, S. (2018). "Refaire Société par la création de communautés d'innovation - Le cas des ateliers SpotLAB sur les nouvelles mobilités en régions ». Revue Française de Gestion, 44(272), 85-102.
} 
Pour citer cet article : Cabanes, B. (2021). Oser la conception innovante pour une industrie de la mode et du luxe durable et responsable. Entreprendre \& Innover, 3(3), 42-55. https://doi.org/10.3917/entin.046.0042

l'identité des objets discutés. Enfin, une phase de construction d'une cartographie de stratégies d'innovation potentielles (phase $P$ ) qui rend compte du processus de conception innovante.

\begin{tabular}{|c|c|c|c|}
\hline & Phase K - Knowledge & Phase C - Concepts & Phase P - Proposals \\
\hline $\begin{array}{l}\text { Choix d'un champ } \\
\text { d'innovation à } \\
\text { explorer }\end{array}$ & $\begin{array}{l}\quad \text { Objectifs } \\
\text { - } \quad \text { Etat de l'art et du « non art » } \\
\text { Mutualisation intensive de } \\
\text { connaissances }\end{array}$ & 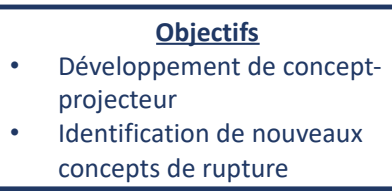 & \begin{tabular}{l}
\multicolumn{1}{c}{ Objectifs } \\
- Cartographie de concepts et \\
de connaissances \\
- Proposition de « roadmaps » \\
de conception innovante
\end{tabular} \\
\hline $\begin{array}{l}\text { Création d'un } \\
\text { groupe de travail }\end{array}$ & $\begin{array}{l}\quad \text { Résultats } \\
\text { - Large ensemble de connaissances } \\
\text { partagées } \\
\text { - Intégration de nouvelles } \\
\text { connaissances } \\
\text { - Restructuration des connaissances } \\
\text { - Identification des stratégies } \\
\text { actuelles }\end{array}$ & $\begin{array}{l}\text { - Ensemble de nouveaux concepts } \\
\text { - Identification de connaissances } \\
\text { manquantes } \\
\text { - Identification de nouvelles voies } \\
\text { d'innovation de rupture } \\
\text { - Remise en question de l'identité } \\
\text { « classique » des objets discutés }\end{array}$ & $\begin{array}{l}\text { - Arborescence de concepts } \\
\text { - Cartographie de stratégies } \\
\text { d'innovations potentielles } \\
\text { - Projet de recherche à poursuivre } \\
\text { - Identification de nouveaux } \\
\text { - } \text { acteurs/partenaires } \\
\text { Preuves de concepts à réaliser }\end{array}$ \\
\hline
\end{tabular}

Les trois phases de la méthode KCP

La mise en place de la méthode a débuté par une phase d'identification des participants à impliquer dans l'atelier KCP. En collaboration avec DEFI Mode (organisme public de soutien et de valorisation du secteur de l'habillement français), une trentaine d'industriels ${ }^{38}$ de la mode et du luxe ont été invités à participer à un atelier KCP d'une demi-journée.

La première phase de l'atelier (phase K) a consisté à soutenir un apprentissage collectif sur la thématique du développement durable et de l'industrie de la mode et du luxe. Dans un premier temps, les participants ont donc été invités à partager leurs expériences sur cette thématique et à identifier différentes bases de connaissances sur le sujet (figure ci-dessous). L'équipe de chercheurs de la chaire $\mathrm{TMCl}$ a effectué

38 Parmi lesquels Eram, LVMH, Manufacture de mode, Euveka, Gorfoo, De Rigueur, Borlis, Les Ateliers Grandis, La Textilerie, Groupe Beaumanoir, etc. 
Pour citer cet article : Cabanes, B. (2021). Oser la conception innovante pour une industrie de la mode et du luxe durable et responsable. Entreprendre \& Innover, 3(3), 42-55. https://doi.org/10.3917/entin.046.0042

un travail important d'interaction avec les acteurs de l'atelier afin de les encourager à explorer un large ensemble de connaissances différentes: définitions du développement durable, définitions possibles de la mode et du luxe (industrie de l'habillement, type particulier de changement social), identification des impacts négatifs de l'industrie de la mode sur la société (pollution, déchets, désindustrialisation, etc.), analyse des différents procédés de fabrication des vêtements, analyse du rôle des prescripteurs, rôles et missions des institutions publiques, impacts de la réglementation, etc.
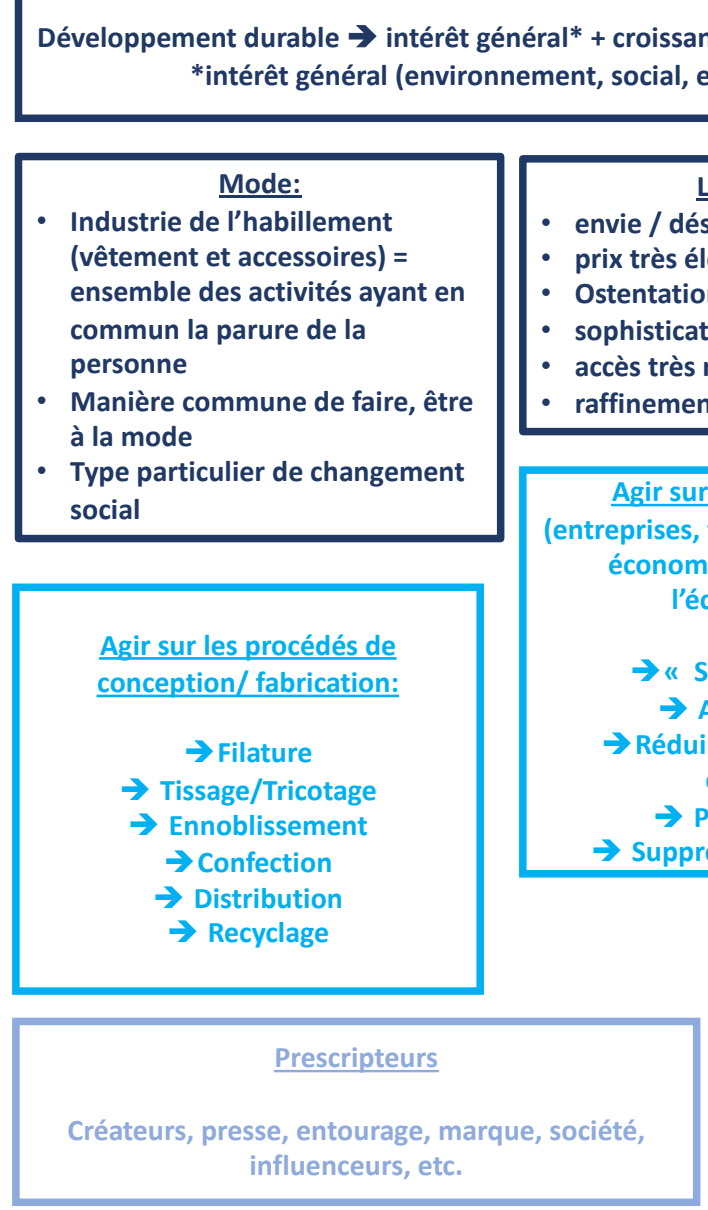

Impacts négatifs de l'industrie de la mode sur la société:

- Pollution de l'air et de l'eau

- Déchets

- Précarité

- Eutrophisation de l'eau

- Absence de recyclage

- Emission de gaz à effet de serre

- Désindustrialisation
- accès très restreint

- raffinement fastueux

Agir sur les institutions (entreprises, fédérations, activités économiques, règles de l'écosystème)

$\rightarrow$ « Slow Fashion »

$\rightarrow$ Anti Fashion

$\rightarrow$ Réduire le rythme des collections

$\rightarrow$ Prescripteurs

$\rightarrow$ Suppression des soldes
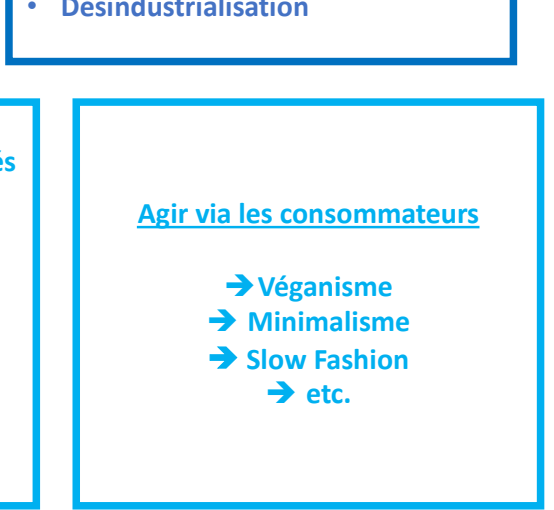

Agir sur la réglementation

$$
\begin{aligned}
& \Rightarrow \text { Loi } \\
& \Rightarrow \text { Quotas } \\
& \Rightarrow \text { Label } \\
& \Rightarrow \text { etc. }
\end{aligned}
$$

Exemple de bases de connaissances sur le développement durable et l'industrie de la mode et du luxe 
Pour citer cet article : Cabanes, B. (2021). Oser la conception innovante pour une industrie de la mode et du luxe durable et responsable. Entreprendre \& Innover, 3(3), 42-55. https://doi.org/10.3917/entin.046.0042

La deuxième phase de l'atelier (phase C) s'est focalisée sur l'élaboration de « concepts projecteurs» afin de faire émerger de nouvelles voies d'innovation. Quatre principaux concepts ont été identifiés afin d'illustrer les différentes approches permettant de traiter les enjeux du développement du durable dans la mode et le luxe : limiter les impacts négatifs, exclure les impacts négatifs, renforcer les impacts positifs, créer de nouveaux impacts positifs. A partir de ces premiers concepts, le groupe de travail a continué à intégrer de nouvelles connaissances (notamment sur les impacts positifs de l'industrie de la mode) et à générer plusieurs branches conceptuelles en ajoutant de nouvelles propriétés. L'approfondissement du concept « limiter les impacts négatifs » a ainsi donné lieu à la création de plusieurs sousconcepts: «limiter les impacts négatifs en agissant sur le cycle de vie des produits », « limiter les impacts négatifs en compensant entièrement les émissions de CO2 », «limiter les impacts négatifs en agissant sur les procédés de fabrication ». Par exemple, le chercheur Marl Liu ${ }^{39}$ propose une solution de rupture particulièrement novatrice: l'introduction de patrons fondés sur une géométrie noneuclidienne. Cette nouvelle façon de concevoir les patrons permet d'éliminer les déchets de matériaux et de réduire considérablement le gaspillage de matière première. Le travail de conception a aussi donné lieu à plusieurs réflexions sur le rôle des labels, afin de certifier la qualité des produits, et sur l'influence des prescripteurs dans la mode (stars de cinéma, sportifs, Instagram, etc.). Le processus de conception innovante a également abouti à la création de concepts visant à rediscuter les codes et le modèle de développement de l'industrie de la mode: « exclure les impacts négatifs en modifiant le rythme des collections », « exclure les

39 Liu, Mark (2017, August). "For a true war on waste, the fashion industry must spend more on research". The Conversation. https://theconversation.com/for-a-true-war-on-waste-the-fashionindustry-must-spend-more-on-research-78673 
Pour citer cet article : Cabanes, B. (2021). Oser la conception innovante pour une industrie de la mode et du luxe durable et responsable. Entreprendre \& Innover, 3(3), 42-55. https://doi.org/10.3917/entin.046.0042

impacts négatifs en supprimant les soldes », notamment pour limiter les effets négatifs de la surconsommation. Enfin, en se focalisant sur l'étude de l'impact positif de l'industrie de la mode sur la santé, l'économie et le tourisme, plusieurs concepts originaux ont émergé : « la mode qui guérit », « la mode qui instruit et qui éduque », « la mode qui permet la cohésion sociale », «la mode qui contribue à l'égalité homme/femme ».

La troisième phase de l'atelier (phase P) a consisté à élaborer une cartographie de des «concepts-projecteurs ${ }^{40}$ » pour mettre en évidence plusieurs stratégies d'innovations potentielles. La cartographies C-K obtenue (figure ci-dessous) ne correspondent pas à un travail d'exploration exhaustif, mais a pour vocation de rendre compte du processus de conception innovante effectué.

40 Objet inconnu qui présente des propriétés désirables pour le concepteur. 
Pour citer cet article : Cabanes, B. (2021). Oser la conception innovante pour une industrie de la mode et du luxe durable et responsable. Entreprendre \& Innover, 3(3), 42-55. https://doi.org/10.3917/entin.046.0042

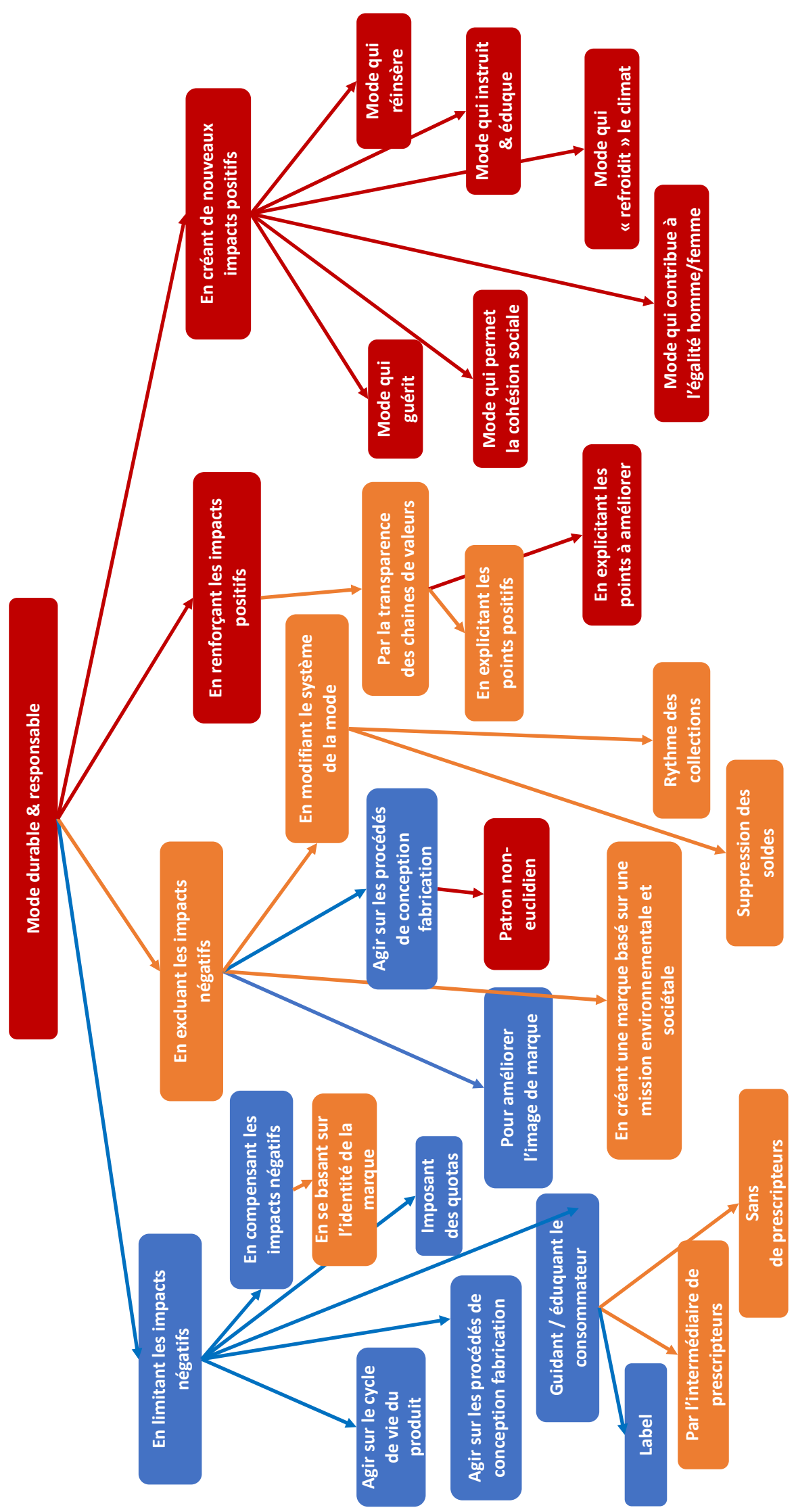

Cartographie C-K : une industrie de la mode et du luxe durable et responsable 
Pour citer cet article : Cabanes, B. (2021). Oser la conception innovante pour une industrie de la mode et du luxe durable et responsable. Entreprendre \& Innover, 3(3), 42-55. https://doi.org/10.3917/entin.046.0042

Les concepts identifiés (en bleu les concepts les moins innovants, et en rouge les concepts les plus en rupture) ne renvoient pas à des solutions immédiates qu'il s'agirait d'appliquer instantanément. Ils constituent des pistes d'exploration et de réflexion dont la valeur et les connaissances associées sont encore à définir et à préciser. Certains concepts se transformeront en objectifs stratégiques et d'autres n'aboutiront à aucune solution concrète. Cependant, l'activité d'invention de concepts et de création de nouvelles connaissances a certainement le mérite de contribuer à l'élaboration d'une démarche d'exploration inédite et à un mode d'apprentissage collectif. La cartographie C-K permet donc de mettre en évidence et d'organiser un large espace d'innovation. Elle agit comme un outil puissant d'aide à la décision stratégique.

Lors de cette recherche-intervention, le rôle des chercheurs-animateurs a été d'accompagner les participants dans l'utilisation de la méthode KCP, mais aussi de soutenir la génération d'idées en proposant des critiques constructives et en catégorisant les idées proposées par thématiques. Cette démarche de conception innovante a suscité une forte appétence des participants, qui ont tout autant apprécié l'esprit de la méthode que la richesse et la diversité des résultats obtenus. Sur le plan cognitif, la méthode KCP a permis, par le biais d'un apprentissage collectif, de structurer un grand nombre de connaissances sur les enjeux du développement durable propres à l'industrie de la mode et du luxe. De plus, notre analyse montre comment une approche fondée sur la conception innovante ex ante a été un support efficace garantissant la création de nouvelles stratégies d'innovations et permettant aux acteurs d'identifier de nouveaux espaces de valeur. Sur le plan organisationnel, 
Pour citer cet article : Cabanes, B. (2021). Oser la conception innovante pour une industrie de la mode et du luxe durable et responsable. Entreprendre \& Innover, 3(3), 42-55. https://doi.org/10.3917/entin.046.0042

la méthode KCP s'est aussi révélée être un outil puissant permettant de fédérer les acteurs de différentes entreprises autour d'enjeux d'innovation communs. Le développement durable en tant que nouveau champ d'innovation devient ainsi un enjeu de conception innovante pour l'ensemble de la filière industrielle de la mode et du luxe. Cette nouvelle perspective encourage ainsi l'ensemble des parties prenantes à développer de nouvelles collaborations dans un esprit de coopétition ${ }^{41}$. Enfin, cette recherche-intervention permet de mettre en évidence un outil opérationnel capable d'organiser l'action collective en vue d'explorer des champs d'innovation inédits.

${ }^{41}$ Collaboration entre différents acteurs économiques qui, par ailleurs, sont des concurrents. Dagnino, G. B., Le Roy, F., \& Yami, S. (2007). « La dynamique des stratégies de coopétition ». Revue française de gestion, (7), 87-98. 